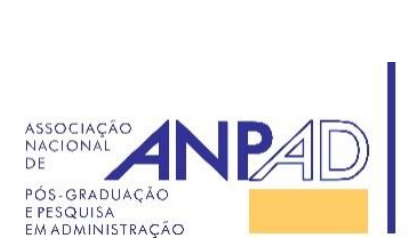
Disponível em
http://www.anpad.org.br/rac
RAC, Rio de Janeiro, v. 21, n. 4, art. 3,
pp. 481-499, Julho/Agosto, 2017
http://dx.doi.org/10.1590/1982-7849rac2017160152
$(\mathrm{cc}) \mathrm{EY}$

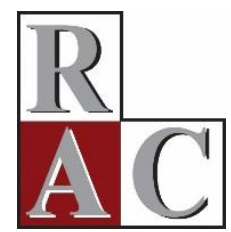

\title{
A Influência do Gênero no Processo Decisório: O Jogo do Ultimato
}

\author{
Gender Influence in the Decision-Making Process: The Ultimatum Game
}

\author{
Angela Cristiane Santos Póvoa ${ }^{1}$ \\ Maickel Robert Maffezzolli ${ }^{1}$ \\ Wesley Pech ${ }^{1,2}$ \\ Wesley Vieira da Silva ${ }^{1}$
}

Pontifícia Universidade Católica do Paraná ${ }^{1}$ Wofford College ${ }^{2}$

Artigo recebido em 06.06.2016. Última versão recebida em 20.01.2017. Aprovado em 20.01.2016. Publicado online em 11.04.2017. 


\title{
Resumo
}

Este artigo analisa as diferenças de gênero no processo decisório por meio de uma abordagem metodológica ainda pouco utilizada no Brasil, embora reconhecida pela academia internacional: o experimento econômico, mais especificamente o Jogo do Ultimato, que pertence ao escopo da Teoria dos Jogos. A condução desse experimento envolveu 320 participantes divididos em 5 subgrupos moderados pelo gênero. Os resultados mostraram que, no processo de interação entre indivíduos, a função utilidade deve ser compreendida não apenas com base na premissa da maximização dos resultados econômicos, mas levar em conta as preferências sociais do indivíduo. Assim, um dos principais achados foi identificar maior propensão masculina à rejeição de propostas advindas de mulheres, não obstante serem essas propostas comparativamente mais generosas. Além disso, foi observado que as mulheres tendem a ser mais generosas em suas ofertas em comparação aos homens. Tais achados são inéditos para o Brasil e podem ser um indicador da influência do gênero sobre o processo decisório, com importantes repercussões para o ambiente organizacional.

Palavras-chave: jogo do ultimato; gênero; tomada de decisão.

\begin{abstract}
This paper analyzes gender differences regarding the decision-making process through a methodological approach, which is still insufficiently used in Brazil. However, it is recognized by the international academy: the economic experiment, specifically the Ultimatum Game, included within the scope of Game Theory. The experiment involved 320 participants divided into 5 subgroups moderated by gender. The results showed that in the process of interaction between individuals, the utility function must be understood not only as based on the premise of maximizing economic results, but it should take into account individual social preferences. Thus, one of the main findings was the identification of a major male predisposition for rejection of proposals coming from women, despite being comparatively more generous. Furthermore, women were observed to be more generous in their offerings compared with men. These finding are pioneering in Brazil and can be considered as an indicator of the influence gender has in the decision-making process, with important implications for the organizational environment.
\end{abstract}

Key words: ultimatum game; gender; decision-making. 


\section{Introdução}

A diversidade de gênero e sua repercussão para o ambiente organizacional têm sido objeto de grande debate acadêmico. Charness e Gneezy (2012) defendem que homens e mulheres diferem na forma como reagem frente às situações que envolvem risco e incerteza em razão das emoções afetarem a avaliação dos resultados de forma diferente para cada gênero. Para Blau e Kahn (2000), embora a distância entre os salários de homens e mulheres venha sendo reduzida desde 1990, esta ainda permanece e, possivelmente, não irá desaparecer, em razão das especificidades de cada gênero.

O tema é polêmico e, dada sua relevância, a literatura acadêmica apresenta numerosos estudos que analisaram essa questão (Castillo \& Cross, 2008; Charness \& Gneezy, 2012; Croson \& Gneezy, 2009; Dittrich, 2015; Eckel \& Grossman, 1998, 2008; Fabre, Causse, Pesciarelli, \& Cacciari, 2015, 2016; García-Gallego, Georgantzis, \& Jaramillo-Gutiérrez, 2012; McGee \& Constantinides, 2013; Meier-Pesti \& Penz, 2008; Ortmann \& Tichy, 1999; Rapoport \& Chammah, 1965; Saad \& Gill, 2001; Solnick, 2001). Em comum, todos esses estudos investigaram as diferenças de gênero a partir do uso de abordagens metodológicas baseadas na economia experimental, mais especificamente, na forma de jogos.

A investigação em torno dessa questão é relevante para o campo da administração, na medida em que aponta caminhos para a gestão do processo decisório, tendo em vista as características mais presentes em cada gênero. Se, de fato, há diferenças sistemáticas na forma como homens e mulheres tomam decisões, então o gênero pode ser apontado como um dos fatores a serem considerados pela gestão corporativa na delegação de funções que exijam a tomada de determinados tipos de decisão. $\mathrm{O}$ desconhecimento acerca dessas influências pode trazer repercussões para o contexto organizacional, uma vez que as implicações decorrentes da diversidade de gênero deixam de ser consideradas ou reconhecidas.

Este artigo analisa a influência do gênero para o processo decisório, e utiliza o escopo da Teoria dos Jogos, mais especificamente o Jogo do Ultimato (JU), como método de investigação. Nesse jogo, o primeiro jogador (proponente) faz uma proposta para a divisão de um determinado valor, e cabe ao segundo jogador (respondente) aceitar ou rejeitar essa proposta. Se o respondente aceitar, o valor é dividido conforme o proposto pelo proponente. Caso o respondente rejeite a proposta, ambos os jogadores nada recebem.

A utilização de Jogos como estratégia metodológica tem como objetivo lançar luz sobre a interação humana em situações de simulação, sendo os Jogos entendidos como modelos simplificados da realidade (Camerer, 2003; Damme et al., 2014). Para Fabre, Causse, Pesciarelli e Cacciari (2016), o JU tem proporcionado um meio eficiente para avaliar o papel das preferências sociais no processo decisório.

A pergunta norteadora desse trabalho, então, foi assim definida: $O$ gênero é fator de influência para as decisões do JU?

Para responder a essa questão, foi proposto um experimento no qual o gênero foi tratado como variável moderadora. Foram criados 5 subgrupos de tratamento, num total de 320 participantes. A única informação disponibilizada aos jogadores foi o gênero do outro jogador, com exceção dos respectivos aos integrantes do grupo de controle. A condução do estudo utilizou uma abordagem do tipo between subjects, com protocolo de condução estabelecido a priori, tendo este o intuito de minimizar o viés do experimentador.

Embora a Teoria dos Jogos, e, mais especificamente, o JU, sejam bastante explorados pela academia internacional como método de pesquisa, no Brasil esse tipo de estudo é ainda pouco frequente, $\mathrm{e}$, por não terem sido identificados trabalhos semelhantes em termos de propósito e metodologia, o presente estudo pode ser considerado pioneiro para o caso brasileiro. Até onde se sabe, não há razão que desaconselhe o uso desse tipo de abordagem metodológica para o Brasil, tendo em vista que a utilização 
de jogos experimentais vem se mostrando adequada e aplicável para as mais diversas culturas e sociedades, sendo possível encontrar estudos conduzidos em países como EUA, Eslovênia, Japão e Canadá (Roth, Prasnikar, Fujiwara, \& Zamir, 1991; Saad \& Gill, 2001), como também em comunidades mais primitivas, como as tribos dos Machiguengas, na Amazônia Peruana, e dos Mapuches, no Chile (Henrich et al., 2001). Güth e Kocher (2014) ressaltam que toda uma geração de economistas comportamentais e experimentais tem utilizado o JU como estratégia de pesquisa e que milhares de estudos foram conduzidos com base nesse Jogo.

O presente trabalho oferece algumas contribuições para o tema investigado, dentre as quais, citam-se: (a) aborda a questão do gênero numa perspectiva experimental, baseada na Teoria dos Jogos, sendo esse tipo de pesquisa algo inovador para o Brasil; (b) contribui para uma maior compreensão acerca do processo decisório moderado pelos gêneros, uma vez que analisa como estes se comportam quando colocados em situação idêntica de decisão; (c) permite a identificação (caso exista) de padrões de decisão associados a cada gênero; (d) avalia como se comportam os gêneros quando em interação com pessoas do mesmo gênero ou de gênero distinto; (e) coloca o Brasil no circuito das pesquisas relativa à Teoria dos Jogos Experimentais, de forma que os resultados aqui descritos poderão somar-se à literatura internacional sobre o tema.

Para o alcance do objetivo proposto por esse trabalho, o presente artigo está estruturado em cinco seções. Após a introdução, segue-se a revisão da literatura, com o desenvolvimento das hipóteses a serem testadas. Na sequência, apresentam-se procedimentos metodológicos, resultados e considerações finais.

\section{A Economia Comportamental e o Jogo do Ultimato}

A economia comportamental (EC) pode ser definida como o estudo das influências cognitivas, sociais e emocionais sobre o comportamento econômico das pessoas (Ávila \& Bianchi, 2015). Essa área do conhecimento ganhou notável impulso a partir dos estudos de Kahneman e Tversky (1979), que desenvolveram a Teoria dos Prospectos e ofereceram importantes contribuições acerca dos fatores que orientam os agentes em decisões que envolvem risco e incerteza. A EC emprega a experimentação como estratégia metodológica para desenvolver teorias sobre como as pessoas tomam decisões (Ávila \& Bianchi, 2015). Assim, a economia experimental é parte integrante da economia comportamental. O experimentador submete indivíduos a decisões reais e não apenas a hipotéticas. $\mathrm{O}$ uso dos experimentos como estratégia metodológica permite realizar inferências acerca do comportamento dos agentes, lançando luz sobre elementos influenciadores do processo decisório que, muitas vezes, não são reconhecidos ou considerados pelos agentes (Crawford, 2002). Eckel e Grossman (2008) esclarecem que a economia comportamental se diferencia da tradicional por considerar os efeitos sistemáticos de fatores como raça e gênero sobre o processo decisório. Nessa perspectiva, o experimento em formato de jogo ajuda a explicar o comportamento de decisão do indivíduo em interação com outros.

O JU pode ser entendido como um modelo simples de tomada de decisão envolvendo barganha, que pertence ao escopo da Teoria dos Jogos. Nesse Jogo, uma determinada quantia monetária deve ser repartida entre dois jogadores. Formalmente, define-se $\mathrm{X}$ como o proponente e $\mathrm{Y}$ como o respondente. $\mathrm{X}$ recebe o valor monetário $p$, e faz uma oferta $x \in[0, p]$ para Y. Essa oferta implica que $\mathrm{X}$ está fazendo a proposta $(x, p-x)$ para Y. Após receber a proposta, Y pode aceitá-la $\delta(x, p-x)=1$, ou rejeitá-la $\delta(x, p$ $x)=0$. Sob o pressuposto de preferências puramente egoístas, o equilíbrio de Nash, perfeito em subjogos, está em o proponente oferecer o menor valor positivo possível e o respondente aceitar essa oferta. A intuição para esse resultado é simples: um respondente puramente egoísta irá sempre aceitar uma oferta positiva, pois qualquer valor monetário positivo é superior a nada receber. Outro proponente, também puramente egoísta, ao antecipar que todas as ofertas positivas serão aceitas, irá maximizar os seus próprios resultados monetários se ele ofertar o menor valor positivo possível (Camerer \& Thaler, 1995; Güth, Schmittberger, \& Schwarze, 1982). No entanto, essa previsão nunca foi observada em estudos experimentais do JU. Na grande maioria das vezes, a oferta média fica entre $40 \%$ e $50 \%$ do valor 
monetário inicial (a oferta modal é quase sempre de 50\%). Respondentes que observam essas ofertas quase sempre as aceitam, mas, quando uma oferta abaixo de $20 \%$ do valor inicial é realizada, muitos respondentes decidem rejeitá-la.

Kahneman, Knetsch e Thaler (1986a) também observou que os montantes mínimos aceitáveis no JU foram em torno de $20 \%$ a $26 \%$ do valor inicial, e que $76 \%$ das recusas foram mais motivadas por sentimentos de justiça do que de avareza, sendo a recusa uma forma de punir uma oferta considerada injusta. Tais resultados foram corroborados por Roth, Prasnikar, Fujiwara e Zamir. (1991) ao conduzirem o JU na Eslovênia, em Jerusalém, nos Estados Unidos e no Japão. Os resultados obtidos pela economia comportamental por meio do JU questionam alguns dos pressupostos da economia neoclássica que preveem a maximização da função utilidade com base na perspectiva dos ganhos econômicos e apontam para a importância das preferências sociais do indivíduo no processo decisório.

O formato desse Jogo é o de interesse para pesquisadores da área comportamental por, pelo menos, duas razões: (a) fornece um teste simples para avaliar se as pessoas estão dispostas a sacrificar algum valor financeiro para reduzir a desigualdade ou para punir um comportamento considerado injusto; (b) testa se as pessoas têm a capacidade de antecipar quais estão dispostas a aceitar ofertas mais ou menos equitativas. Do ponto de vista metodológico, ele possui as vantagens de ser simples, de fácil implementação e, por ser um jogo sequencial com apenas duas pessoas, cria um ambiente mais controlado do ponto de vista metodológico.

\section{As diferenças entre os gêneros e o processo decisório}

Distintas áreas do conhecimento têm em comum o gênero como variável de interesse. Nos campos da psicologia e da neurologia, são observados resultados consistentes que apontam para a superioridade feminina no que tange as habilidades verbais, ao passo que, para os homens, as habilidades espaciais e aritméticas tendem a ser mais proeminentes (Ardila, Rosseli, \& Inozemtseva, 2011; Weiss, Kemmlera, Deisenhammerb, Fleischhackera, \& Delazer, 2003). Na área da comunicação, a linguagem masculina tende a ser caracterizada por atributos como objetividade, concisão e instrumentalidade, enquanto que, para as mulheres, a comunicação tende a ser vista como mais elaborada, indireta e afetiva (Maltz \& Borker, 1982; Mulac, Bradac, \& Gibbons, 2001). Também Beckmann e Menkhoff (2008) e Prime, Carter e Welbourne (2009) afirmam que há diferenças robustas entre homens e mulheres no que se refere a fatores como predisposição ao risco, confiança excessiva, comportamento competitivo e liderança. Tais diferenças mostram-se especialmente relevantes para o campo da administração.

A literatura dedicada ao estudo da diversidade de gênero tem se utilizado em larga medida da abordagem metodológica propiciada pela Teoria dos Jogos, mais especificamente, do JU (Croson \& Gneezy, 2009; Eckel \& Grossman, 1998, 2008; Fabre et al., 2015, 2016; McGee \& Constantinides, 2013; Saad \& Gill, 2001; Solnick, 2001; Zak et al., 2009). Nesse tipo de abordagem, os montantes ofertados por cada gênero ao interagirem com pessoas do mesmo gênero ou de gênero diferente, bem como a decisão de aceitar ou de recusar as propostas do jogo, são variáveis de interesse para o pesquisador pelo seu potencial para revelar aspectos importantes e, muitas vezes, não explícitos no contexto do processo decisório (Camerer, 2003; Svorenčík \& Mass, 2016).

Solnick (2001), em estudo conduzido junto a estudantes da Universidade da Pennsylvania (EUA), analisou o comportamento de homens e mulheres no JU por meio de dois tratamentos: no primeiro, o gênero dos jogadores era anônimo, ao passo que, no segundo, era de conhecimento comum. Foi observado que o gênero do jogador 1 (ou proponente) não era fator de influência para o montante ofertado, contudo o mesmo não se mostrou verdadeiro para o jogador 2 (respondente), uma vez que as ofertas mais generosas foram direcionadas aos homens. Também foi observado que os jogadores proponentes de ambos os gêneros mostravam-se mais exigentes em relação as ofertas femininas, sendo esperado um montante mínimo mais elevado para aceite do valor proposto por jogadoras. Também, as maiores taxas de rejeição foram observadas em jogos conduzidos somente entre mulheres.

Em estudo semelhante, conduzido por Eckel e Grossman (2001), foi identificado que as propostas femininas foram, em média, mais generosas que as masculinas, independente do gênero do jogador 
respondente, e que as mulheres foram mais propensas a não recusarem as ofertas do jogo. Além disso, as ofertas advindas de mulheres tendiam a ser menos rejeitadas, sendo esse resultado interpretado como uma forma de cordialidade para com esse gênero. Tanto Solnick (2001) quanto Eckel e Grossman (2001) observaram que as ofertas direcionadas aos homens eram, em média, mais elevadas do que as direcionadas às mulheres. Também Saad e Gill (2001), em estudo conduzido no Canadá, utilizaram o JU em rodada única numa abordagem evolucionária, e também identificaram que as ofertas masculinas tendiam a ser mais generosas quando o outro jogador era mulher.

Nessa perspectiva, tanto os montantes propostos no JU quanto as decisões relativas ao aceite ou à recusa das propostas apresentaram resultados que reforçaram a importância do gênero para as respostas do Jogo. Essa afirmação pode ser analisada à luz dos achados de Güth, Schmittberger e Schwarze (1982) e Kahneman, Knetsch e Thaler (1986b), que conduziram o JU sem o tratamento do gênero como variável moderadora, em comparação aos estudos conduzidos por Solnick (2001); Eckel e Grossman (2001); Saad e Gill (2001), que tinham o gênero como variável moderadora.

Segundo a perspectiva da EC, sendo o Jogo um modelo simplificado de decisão, os achados desse tipo de experimento podem lançar luz sobre fatores que exercem influência sobre o processo decisório, sendo esse aspecto de especial interesse para o campo da administração.

Dentre os fatores mais comumente apontados como explicativos para as diferenças nos resultados do JU quando moderados pelo gênero, citam-se a aversão ao risco (Charness \& Gneezy, 2012; Croson \& Gneezy, 2009; Eckel \& Grossman, 1998, 2008), o altruísmo (Andreoni \& Vesterlund, 2001), o posicionamento diferenciado em situações de barganha (Castillo \& Gross, 2008; Solnick, 2001); os estereótipos de gênero (Fabre et al., 2016; Kray \&Thompson, 2005), entre outros.

Para Croson e Gneezy (2009), um dos fatores associados à influência do gênero nos resultados do JU é a maior aversão ao risco por parte do gênero feminino, o que induziria a uma oferta mais elevada no Jogo. Nessa perspectiva, o perfil masculino seria, em geral, mais propenso a lidar com situações que exigem maior tolerância ao risco. Para García-Gallego, Georgantzis e Jaramillo-Gutiérrez (2012), a maior disposição das mulheres em aceitarem ofertas mais baixas pode estar relacionada a uma maior expectativa desse gênero para alcançar resultados mais pessimistas quando comparados aos alcançados por homens, sendo essa expectativa alimentada pelo fato de que, em muitos países, as mulheres têm superado uma longa história de discriminação no ambiente de trabalho.

Para Solnick (2001), as posturas assumidas por cada gênero quando em situação de barganha podem explicar os diferentes resultados econômicos obtidos no JU. Castillo e Gross (2008) acreditam que tais posturas são potencialmente explicativas para os salários mais altos recebidos por homens e estão relacionadas aos processos de barganha que ocorrem entre a empresa e o funcionário no momento da contratação e, mais tarde, ao longo de sua carreira. Nesse processo, as mulheres tendem a aceitar ofertas menores.

Andreoni e Vesterlund (2001) acreditam que o altruísmo está relacionado aos diferentes resultados envolvendo gêneros no JU, sendo observado que o comportamento masculino tende a ser menos altruísta quando o processo decisório envolve valores financeiros mais elevados, ao passo que as mulheres tendem a ser menos altruístas quando há valores mais baixos em disputa. Nessa perspectiva, a demanda masculina mostra-se mais sensível ao preço.

A interação entre os gêneros também tem se mostrado relevante para o processo decisório. Sutter, Bosman, Kocher e Winden (2009) observaram que em JU, o pareamento entre pessoas do mesmo gênero é motivo de reações que apontam para a existência de maior competitividade e retaliação, e, consequentemente, menor eficiência. Já para Gneezy, Niederle e Rustichini (2003), existe maior competitividade quando há interações entre os diferentes gêneros em comparação com competições entre indivíduos do mesmo gênero.

Güth e Kocher (2014) lembram que "enquanto a estrutura do JU é uma das mais simples que pode ser imaginada (dois jogadores, dois estágios, informações completas), as motivações que orientam as decisões dos jogadores são diversas, e isto tem tomado tempo dos pesquisadores para compreendê-las"

RAC, Rio de Janeiro, v. 21, n. 4, art. 3, pp. 481-499, Julho/Agosto, 2017, www.anpad.org.br/rac (cc)) 
(p. 397). Dessa forma, é importante lembrar que o presente estudo tem como objetivo analisar a influência do gênero para os resultados do JU para o caso brasileiro, buscando lançar luz sobre a relevância (ou não) do fator gênero para o processo decisório e suas potenciais repercussões para a gestão organizacional. Contudo, não faz parte do escopo desse estudo a investigação acerca dos motivos que orientaram as decisões por parte dos jogadores, o que demandaria estudos adicionais.

Nessa perspectiva, tomando como base a revisão da literatura, foram propostas duas hipóteses de trabalho que avaliaram a influência do gênero para as decisões relativas ao JU:

H1: Proponentes femininas ofertam de maneira mais generosa em comparação aos proponentes masculinos.

H2: Respondentes masculinos tendem a aceitar mais as ofertas advindas de mulheres em comparação com as ofertas advindas de homens.

\section{Procedimentos Metodológicos}

Como já mencionado, este estudou adotou o experimento baseado na Teoria dos Jogos como estratégia de pesquisa, e, de forma mais específica, o JU. Em um ambiente controlado, a saber, uma sala de aula reservada para esse fim, foram reunidos os indivíduos que aceitaram participar do experimento. Foram convidados estudantes de uma universidade de grande porte no Estado do Paraná como sujeitos da pesquisa. A amostra foi composta por 169 homens e 151 mulheres pertencentes aos cursos de economia, contabilidade, turismo, marketing e administração de empresas. Destes, 289 indicaram seu estado civil como solteiro, o que representou $89,7 \%$ da amostra. O exercício de atividade remunerada foi identificado em cerca de $75 \%$ dos estudantes. A idade média foi igual a 21,3 anos. Os participantes foram recrutados por meio de convite pessoal durante o período de aulas e por e-mails duas semanas antes da data marcada. A adesão e a participação no experimento foram voluntárias e os convites informavam sobre a possibilidade de ganho financeiro sem menção do valor, o que caracterizou uma amostra por conveniência. Em geral, os valores monetários oferecidos em estudos dessa natureza permeiam US $\$ 10,00$. Em condição de aproximação, foi proposto o valor de $\mathrm{R} \$ 14,00$ para cada dupla de jogadores.

Na data marcada, coube ao experimentador explicar o experimento para todos os participantes, e, uma vez que o experimento era do tipo between subjects e deveria ocorrer repetidas vezes com diferentes participantes até alcançar o número previsto de 300 indivíduos, foi previamente elaborado um roteiro de aplicação do Jogo como forma de possibilitar igual aplicação do experimento nas diferentes rodadas, minimizando (mas não eliminando) o viés do experimentador. Antes da aplicação do experimento, foram realizados pré-testes tanto do roteiro de fala do experimentador como da forma de condução do experimento, buscando assegurar o entendimento dos participantes acerca das regras do Jogo. Também foi solicitado que, durante o experimento, os participantes não interagissem com outras pessoas e não utilizassem nenhum tipo de aparelho eletrônico.

Assim, todas as regras e elementos do Jogo eram de conhecimento comum (informação completa) entre todos os participantes, que tiveram a garantia do sigilo de suas identidades. Cada um preencheu uma breve ficha com seus dados sociodemográficos (nome, gênero, faixa de renda familiar, experiência profissional, estado civil, idade, nível de escolaridade com indicação dos cursos e telefone para contato). Straits e Singleton (2010) relatam que o sucesso de um experimento está na capacidade do experimentador em reduzir potenciais vieses que possam distanciar o experimento da realidade que está sendo simulada em laboratório. Dessa forma, o estudo foi conduzido com o efetivo pagamento da quantia acertada entre os jogadores.

Os participantes receberam, além de instruções orais, instruções escritas, bem como um formulário para a decisão acerca do Jogo. Na primeira rodada, o jogador denominado 1 recebeu uma ficha na qual ele tinha a opção de fazer a oferta, ou seja, propor a divisão do valor tendo em vista o 
montante de $\mathrm{R} \$ 14,00$ (quatorze reais). $\mathrm{O}$ aceite da proposta foi condicionado ao próximo jogador, denominado jogador 2. Isso implica em compreender que este Jogo foi conduzido em três momentos: o da oferta, pelo jogador 1 (proponente); o do aceite/recusa, pelo jogador 2 (respondente); e o retorno da decisão do jogador 2 para o jogador 1, com o pagamento dos valores acordados, quando fosse o caso. $\mathrm{O}$ proponente só tinha direito a receber sua parte na divisão caso o respondente aceitasse a proposta.

Embora haja certa crítica em relação à condução de experimentos com estudantes sob a alegação de que não são representativos da população, para Güth e Kocher (2014) este é um grupo mais homogêneo em termos de suas características socioeconômicas, o que favorece a redução dos vieses da amostra. Exadaktylos, Espín e Brañas-Garza (2013), ao conduzirem experimentos baseados em jogos econômicos com amostras formadas por estudantes e não estudantes, identificaram que os estudantes formam um grupo apropriado para a condução de pesquisas baseadas em jogos experimentais. Além disso, a literatura relativa ao JU é predominantemente composta por estudos que utilizaram estudantes como sujeitos de pesquisa, o que favorece a comparação dos resultados obtidos no Brasil e no exterior.

\section{Gênero como variável moderadora}

Para a presente pesquisa, foram criados 5 grupos de tratamento de acordo com as possíveis interações entre as duplas de jogadores, conforme Tabela 1. A quantidade mínima de casos prevista para cada célula de coleta foi de 60 indivíduos (30 pares), tendo em vista a estatística T-Student.

Tabela1

\section{Grupos de Tratamento Moderados pelo Gênero}

\begin{tabular}{lllc}
\hline Estrutura dos grupos & Proponente & Respondente & $\begin{array}{c}\text { Quantidade mínima de } \\
\text { pares }\end{array}$ \\
\hline Grupo & Mulher & Mulher & 30 \\
MH & Mulher & Homem & 30 \\
HH & Homem & Homem & 30 \\
HM & Homem & Mulher & 30 \\
Controle & Homem ou mulher & Homem ou mulher & 30 \\
\hline
\end{tabular}

Nota. Fonte: Elaborada pelos autores.

A premissa metodológica deste estudo previa o controle do gênero dos jogadores, e, por esse motivo, essa informação era de conhecimento comum entre eles, com exceção do grupo de controle. É importante ressaltar que a quantidade de participantes definida para este experimento obedeceu ao observado em estudos semelhantes conduzidos no exterior, a exemplo de Solnick, (2001) e Eckel e Grossman (2001).

\section{Resultados do Experimento}

Ao final do experimento, foram contabilizados 320 participantes, conforme Tabela 2. Embora não tenham sido designados para cada grupo de tratamento de forma aleatória, tendo em vista os propósitos do experimento, os participantes do Jogo apresentaram um padrão semelhante em relação aos aspectos sociodemográficos. Essa avaliação é relevante porque reduz os vieses amostrais entre os grupos. 
Tabela 2

Quantidade de Participantes

\begin{tabular}{ccccc}
\cline { 2 - 5 } & Total & Percentual & Proponentes & Respondentes \\
\hline MM & 62 & 19.3 & 31 & 31 \\
HH & 68 & 21.1 & 34 & 34 \\
Controle & 60 & 19.3 & 30 & 30 \\
HM & 68 & 21.1 & 34 & 34 \\
MH & 62 & 19.3 & 31 & 31 \\
Total & 320 & 100.0 & 160 & 160 \\
\hline
\end{tabular}

Nota. Fonte: Dados da pesquisa. $\mathrm{M}=$ Mulher $/ \mathrm{H}=$ Homem / Controle = o gênero dos jogadores era anônimo nesse grupo.

A Tabela 3 apresenta a estatística descritiva dos valores ofertados pelos proponentes, tendo em vista cada grupo de tratamento.

Tabela 3

Comportamento dos Proponentes do Jogo em Relação ao Valor Ofertado

\begin{tabular}{cccccc}
\cline { 2 - 6 } & MM & HH & MH & HM & Controle \\
\hline Média & 7.14 & 6.55 & 7.29 & 6.94 & 6.91 \\
Mediana & 7 & 7 & 7 & 7 & 7 \\
Moda & 7 & 7 & 7 & 7 & 7 \\
Mínimo & 5 & 2 & 5 & 4 & 5 \\
Máximo & 14 & 9 & 8 & 14 & 10 \\
Desv. Padrão & 1.5041 & 1.2108 & 0.8132 & 2.029 & 1.120 \\
\hline
\end{tabular}

Nota. Fonte: Dados da pesquisa. $\mathrm{M}=$ Mulher $/ \mathrm{H}=$ Homem $/$ Controle $=\mathrm{o}$ gênero era desconhecido pelos jogadores.

Segundo os dados observados, o valor mais frequentemente ofertado pelos proponentes foi igual a $\mathrm{R} \$ 7,00$, o que representou $50 \%$ do valor oferecido no jogo, corroborando a literatura sobre o tema. As maiores médias para os valores ofertados ocorreram nos grupos nos quais as mulheres foram as proponentes (MM e MH). O grupo que apresentou maior desvio padrão foi o HM. Os valores máximos ofertados pelos proponentes ocorreram em grupos nos quais as mulheres eram as respondentes. Já os valores mínimos ofertados ocorreram em grupos nos quais os homens eram os proponentes.

O próximo passo foi analisar se as ofertas realizadas nos grupos que tinham o conhecimento acerca do gênero do outro jogador eram estatisticamente diferentes das observadas no grupo de controle, que não recebeu essa informação. Tendo em vista que o teste de Shapiro-Wilk apontou para a normalidade dos dados, foi conduzido o teste de diferenças de T-Student, conforme Tabela 4.

Tabela 4

Diferença entre Grupos de Gênero Conhecido e Grupo de Controle

\begin{tabular}{lcccccc}
\hline Proposta & N & Média & Erro Padrão & Diff $<\mathbf{0}$ & Diff $=\mathbf{0}$ & Diff $>\mathbf{0}$ \\
\hline Grupo de Controle & 30 & 6,91 & .132 & 0.6071 & .7857 & 0.3929 \\
Demais Grupos & 130 & 6,98 & .156 & & & $\mathrm{~T}=0.2723$ \\
\hline
\end{tabular}

Nota. Fonte: Dados da pesquisa.

RAC, Rio de Janeiro, v. 21, n. 4, art. 3, pp. 481-499, Julho/Agosto, 2017, www.anpad.org.br/rac (cc)) 
O resultado do teste não apontou a existência de diferenças estatisticamente significativas entre os dados das duas amostras. Nesse sentido, as ofertas propostas por homens e mulheres foram, conjuntamente, semelhantes às do grupo de controle.

Assim, tendo em vista que a análise conjunta dos gêneros não se mostrou significativa, o passo seguinte foi analisar se havia diferenças significativas entre as ofertas propostas por homens e por mulheres separadamente. Os grupos foram reorganizados e um novo agrupamento foi testado via Mann Whitney, considerando 2 amostras independentes formadas pelas ofertas femininas versus masculinas. Este teste é similar ao teste $\mathrm{T}$ para amostras independentes, mas, como os dados mostraram-se não paramétricos, ao invés de comparar as variâncias oriundas das médias, foram comparados os ranks (ou postos), calculados a partir das medianas. $\mathrm{O}$ resultado do teste permitiu observar diferenças significativas entre as ofertas de homens e mulheres $(\mathrm{U}=2712,5 \mathrm{p}<0,05)$. Os homens mostraram-se mais dispostos a ofertar valores menores comparados às mulheres, e, por consequência, alcançavam maior resultado individual.

De forma complementar, foi conduzida uma análise que considerou o valor médio das ofertas ( $\mathrm{R} \$ 6,90)$, sendo o mínimo de $\mathrm{R} \$ 0,00$ e o máximo de $\mathrm{R} \$ 14,00$. As ofertas foram categorizadas em injustas (valores entre $\mathrm{R} \$ 0,00$ e $\mathrm{R} \$ 6,00$ ) e justas ou generosas (valores superiores a 6,0). Essa divisão orientou-se pelos achados de Güth e Kocher (2014).

Os resultados apresentados na Tabela 5 permitem observar uma significativa diferença $\left(\chi^{2}=8,384\right.$ $\mathrm{df}=1 \mathrm{p}<0,000)$ entre a proporção de ofertas injustas realizadas pelos homens em comparação com as das mulheres. Das ofertas consideradas injustas, $72 \%$ foram propostas por homens e, dentre as que foram consideradas justas, $54 \%$ foram propostas por mulheres.

Tabela 5

\section{Distribuição de Gênero por Oferta Justa e Injusta}

\begin{tabular}{ccccc}
\cline { 3 - 4 } & & Mulher & Homem & \\
\hline Ofertas Injustas & Casos & 13 & 34 & 47 \\
& $\%$ & $28 \%$ & $\mathbf{7 2 \%}$ & $100,0 \%$ \\
\hline Ofertas Justas & Casos & 61 & 53 & 113 \\
& $\%$ & $\mathbf{5 4 \%}$ & $46 \%$ & $100,0 \%$ \\
\hline
\end{tabular}

Nota. $\left(\chi^{2}=8,384 \mathrm{df}=1 \mathrm{p}<0,000\right)$. Fonte: Dados da pesquisa.

Até esse momento, a análise dos dados permitiu observar que havia diferenças estatisticamente significativas entre as ofertas feitas por homens e por mulheres. Buscando tornar mais robusta a análise dos dados, foi proposto um modelo de regressão que pudesse apontar quais variáveis seriam explicativas para os valores ofertados no Jogo. Nessa regressão, o grupo de controle não foi inserido, tendo em vista que o objetivo era captar os efeitos no montante ofertado quando o gênero era conhecido. Dessa forma, o montante ofertado pelos proponentes foi colocado como variável dependente. As variáveis relativas aos gêneros do proponente e do respondente $\left(D_{1}\right.$ e $D_{2}$ respectivamente) assumiram valores iguais a 0 para homem e 1 para mulher. A variável $\mathrm{D}_{3}$ foi incluída no modelo para captar a possibilidade das pessoas se comportarem de maneira diferente, a depender de sua interação com pessoas do mesmo gênero ou de gêneros diferentes. Para tanto, a variável foi operacionalizada a partir do valor absoluto resultado da subtração das variáveis $\mathrm{D}_{1}$ e $\mathrm{D}_{2}$. Já as variáveis $\mathrm{X}_{4}$ e $\mathrm{X}_{5}$ utilizaram os dados obtidos nos questionários respondidos pelos participantes. O modelo testado foi assim descrito:

$$
\begin{aligned}
& Y=\alpha+\beta_{1} D_{1}+\beta_{2} D_{2}+\beta_{3} D_{3}+\beta_{4} X_{4}+\beta_{5} X_{5}+u_{i} \\
& Y=\text { variável dependente relativa ao valor ofertado pelo proponente } \\
& D_{1}=\text { gênero do proponente (1 para mulher, } 0 \text { para homem) }
\end{aligned}
$$




$$
\begin{aligned}
& D_{2}=\text { gênero do respondente ( } 1 \text { para mulher, } 0 \text { para homem) } \\
& D_{3}=\text { interação entre gêneros ( } 1 \text { se os gêneros foram diferentes e } 0 \text { se eles foram iguais) } \\
& X_{4}=\text { variável escalar para renda do proponente } \\
& X_{5}=\text { variável composta por número de anos (idade) do proponente }
\end{aligned}
$$

Foram analisadas as principais violações para os resultados obtidos pelo modelo de regressão. $\mathrm{O}$ teste VIF (variance inflator fator) não indicou a presença de multicolinearidade nos dados, tendo em visto que o maior valor obtido nessa estatística foi igual a 1,02. Já o teste de Breusch-Pagan e CookWeisberg falhou em rejeitar a hipótese de homocedasticidade dos dados (prob $>$ chi $2=0,116$ ). Para avaliar possíveis problemas de especificação do modelo, foi conduzido o teste de Ramsey, cujo resultado apontou para a não omissão de variáveis relevantes no modelo a um nível de significância de 5\% (prob $>\mathrm{F}=0.095$ ). Por fim, o teste de Hausman mostrou que não havia diferenças significativas na estimação por Mínimos Quadrados Ordinários (MQO) nem por Mínimos Quadrados em Dois Estágios (MQ2E), afastando possíveis problemas relacionados à endogeneidade ( $p$-value < 0,01). Contudo, é importante ressaltar que, em teoria, possíveis problemas de endogeneidade não podem ser completamente descartados tendo em vista a natureza das variáveis dependentes e independentes presentes no modelo. A Tabela 6 apresenta os resultados obtidos pelo modelo de regressão.

Tabela 6

\section{Resultados da Regressão para Oferta do Proponente como Variável Dependente}

\begin{tabular}{lcccc}
\hline Descrição das variáveis & Variáveis do modelo & Coeficientes & $\mathbf{t}$ & p-valor \\
\hline gênero do proponente & $\mathrm{D}_{1}$ & .5516 & 2.06 & $\mathbf{0 . 0 4 2}$ \\
gênero do respondente & $\mathrm{D}_{2}$ & .1575 & 0.63 & 0.529 \\
interação entre gêneros & $\mathrm{D}_{3}$ & .3015 & 1.20 & 0.232 \\
Renda & $\mathrm{X}_{1}$ & -.2443 & -2.18 & $\mathbf{0 . 0 3 1}$ \\
Idade & $\mathrm{X}_{2}$ & .0061 & 0.031 & 0.822 \\
Cons & Cons & 7.042 & 10.05 & 0.000 \\
\hline
\end{tabular}

Nota. $R$-square $=0,1043 / n=130$. Fonte: Dados da pesquisa.

Os resultados mostraram que duas variáveis foram explicativas para o valor ofertado no Jogo: o gênero do proponente e a sua renda. $\mathrm{O}$ gênero feminino mostrou-se mais propenso a ofertar valores mais altos, corroborando Solnick (2001), Saad e Gill (2001) e Eckel e Grossman (2001). Esse achado permitiu aceitar a primeira hipótese deste estudo, a saber: o gênero feminino apresenta maior propensão a ofertar de maneira mais generosa quando comparado ao gênero masculino. Contrariamente ao esperado, os proponentes com menores níveis de renda mostraram-se mais generosos em suas ofertas. A relação entre oferta mais generosa e renda inferior pode, entre outras possibilidades, revelar a aversão ao risco da perda do valor do jogo, o que incentivaria uma divisão mais generosa e com menor chance de rejeição. Contudo, essa questão merece maior aprofundamento.

Tendo em vista que o gênero feminino mostrou-se menos propenso a maximizar o resultado individual, seja por razões de altruísmo (Andreoni \& Vesterlund, 2001), aversão ao risco (Eckel \& Grossman, 2008) ou, ainda, por outras razões a serem investigadas e descobertas, o que se pode afirmar é que a gestão organizacional pode se beneficiar dessa característica feminina de várias maneiras, como, por exemplo, delegando para pessoas desse gênero, cargos ou funções que exijam a tomada de decisões que demandem uma postura mais equitativa, conciliatória ou mesmo conservadora. Já decisões que tenham um caráter mais maximizador e de assunção de risco podem estar mais próximas ao perfil masculino. É importante ressaltar que não se trata de promover uma categorização de funções baseada 
em gênero, mas de reconhecer que o gênero é mais uma variável a ser considerada como relevante na gestão do portfólio de decisões da organização.

Para investigar a segunda hipótese proposta, a saber, homens tendem a aceitar mais as ofertas do Jogo quando estas são de proponentes mulheres, o primeiro passo foi analisar os percentuais de rejeição em cada tratamento do experimento. Os dados da Tabela 7 apontaram para o primeiro indício de que o gênero era fator relevante para a decisão de aceite ou recusa no Jogo.

Tabela 7

Distribuição de Aceite/Recusa da Oferta entre os Grupos

\begin{tabular}{lllllll}
\cline { 3 - 6 } & & \multicolumn{2}{l}{ Grupos } & & & \\
\cline { 3 - 7 } & & MM & HH & Controle & HM & MH \\
\hline Recusa & Casos & 3 & 1 & 1 & 4 & 7 \\
& $\%$ & $9.6 \%$ & $2.9 \%$ & $3.3 \%$ & $11.7 \%$ & $\mathbf{2 3 . 3 \%}$ \\
\hline Aceite & Casos & 28 & 33 & 29 & 30 & 23 \\
& $\%$ & $90.3 \%$ & $97.1 \%$ & $96.7 \%$ & $88.3 \%$ & $76.7 \%$ \\
\hline Total & Casos & 31 & 34 & 30 & 34 & 30 \\
& $\%$ & $100 \%$ & $100 \%$ & $100 \%$ & $100 \%$ & $100 \%$ \\
\hline
\end{tabular}

Nota. Fonte: Dados da pesquisa.

A análise descritiva dos dados mostrou que o maior percentual de rejeição de ofertas pelo jogador 2 ocorreu quando este era homem e o proponente era mulher (MH). Nesse grupo, 23,3\% das ofertas foram rejeitadas. Curiosamente, quando as mulheres foram as respondentes, os percentuais de rejeição não pareceram se modificar em função do gênero dos proponentes, tendo em vista os resultados observados nos grupos MM (9.67\%) e HM (11.7\%). Contudo, quando o Jogo ocorreu somente entre homens, observou-se o menor percentual de rejeição (2.9\%), mostrando uma aparente propensão dos homens a aceitarem propostas de outros homens, sendo esse número igual ao observado no grupo de controle. Buscando compreender se as rejeições observadas nos grupos de tratamento estavam relacionadas ao valor ofertado, foram analisados os valores médios das ofertas que foram recusadas em comparação com a média das ofertas que foram aceitas. Os resultados estão dispostos na Tabela 8.

Tabela 8

Valor Médio Ofertado por cada Grupo de Tratamento

\begin{tabular}{lllllll}
\cline { 3 - 7 } & & \multicolumn{3}{l}{ Grupos de tratamento } & & \\
\cline { 3 - 7 } & & $\mathbf{M M}$ & $\mathbf{H H}$ & Controle & $\mathbf{H M}$ & $\mathbf{M H}$ \\
\hline Recusa & Casos & 3 & 1 & 1 & 4 & 7 \\
& Valor médio & $\mathrm{R} \$ 7.33$ & $\mathrm{R} \$ 2.0$ & $\mathrm{R} \$ 5.0$ & $\mathrm{R} \$ 4.75$ & $\mathbf{R} \$ \mathbf{6 . 8 0}$ \\
\hline Aceite & Casos & 28 & 33 & 29 & 30 & 23 \\
& Valor médio & $\mathrm{R} \$ 7.12$ & $\mathrm{R} \$ 6.69$ & $\mathrm{R} \$ 6.88$ & $\mathrm{R} \$ 6.71$ & $\mathrm{R} \$ 7.19$ \\
\hline
\end{tabular}

Nota. Fonte: Dados da pesquisa.

Os dados da Tabela 8 mostram que a oferta média do grupo que obteve o maior número de rejeição $(\mathrm{MH})$ foi igual a $\mathrm{R} \$ 6.80$, uma média somente inferior à observada no grupo MM. Observa-se também que o grupo HM, no qual as ofertas foram comparativamente menores ( $\mathrm{R} \$ 4.75$ ) não foi o grupo que apresentou o maior percentual de rejeição. Nesse sentido, embora o grupo HM tenha recusado ofertas provavelmente em função do valor ofertado, o mesmo não parece ter ocorrido no grupo MH. A oferta 
recusada no grupo HH pareceu justificada em razão de seu valor ser inferior a $20 \%$ do valor em disputa, o que corrobora a literatura sobre o tema. Fato interessante ocorreu no grupo MM, no qual a média de valor ofertado por mulheres e recusado por mulheres foi maior do que a média ofertada considerando todos os demais grupos (R $\$$ 7.33). Assim, pelo menos duas possibilidades podem ser colocadas: (a) a não compreensão do Jogo por parte dessas jogadoras; ou (b) uma possível propensão à recusa de ofertas generosas advindas de outras mulheres. Esse resultado é incomum e motivou a busca por sua compreensão a partir da literatura sobre o tema. Henrich et al. (2001) apontaram para a possibilidade da recusa de uma oferta generosa ocorrer em razão desta ser compreendida como uma forma de insulto. Contudo, não se pode afirmar que esse seja o caso, o que demandaria estudos adicionais sobre a questão.

A Tabela 9 permite observar diferenças nas respostas relativas ao aceite ou à recusa da oferta quando o jogador 2 é homem e conhece o gênero do proponente, o que ocorreu nos grupos $\mathrm{HH}$ e $\mathrm{MH}$. Com diferença significativa $\left(\chi^{2}=6,059 \mathrm{df}=1 \mathrm{p}<0,05\right)$, é possível observar que os homens tendem a rejeitar mais as ofertas quando estas são propostas por mulheres. Assim, os homens recusaram mais as ofertas do Jogo (87.5\%) quando advindas de mulheres em comparação com as ofertas masculinas (12.5\%). Este achado apontou para uma aparente propensão masculina à rejeição das ofertas femininas.

Tabela 9

Distribuição de Aceite/Rejeição por Grupos HH e MH

\begin{tabular}{lllll}
\cline { 3 - 4 } & & HH & MH & \\
\hline RECUSA & Casos & 1 & 7 & 8 \\
& $\%$ & $12.5 \%$ & $\mathbf{8 7 . 5 \%}$ & $100.0 \%$ \\
\hline \multirow{2}{*}{ ACEITE } & Casos & 33 & 23 & 56 \\
& $\%$ & $\mathbf{5 8 . 9 \%}$ & $41.1 \%$ & $100.0 \%$ \\
\hline \multirow{2}{*}{ Total } & Casos & 34 & 30 & 64 \\
& $\%$ & $53.1 \%$ & $46.9 \%$ & $100.0 \%$ \\
\hline
\end{tabular}

Nota. Fonte: Dados da pesquisa.

Por fim foram testados os fatores potencialmente explicativos para o aceite ou a recusa das ofertas do Jogo. Nesse caso, tendo em vista que a variável dependente era binária (aceite $=1$ e recusa $=0$ ), a análise foi conduzida por meio de uma regressão logística. Esta técnica estatística foi utilizada para complementar a compreensão sobre os fatores que ajudam a explicar o aceite/recusa por parte dos respondentes. As variáveis independentes incluídas no modelo foram o gênero do proponente, o gênero do respondente, a interação entre ambos os gêneros, a renda e a idade dos respondentes. $\mathrm{O}$ modelo foi assim descrito:

$$
\begin{aligned}
& Y=\alpha+\beta_{1} D_{1}+\beta_{2} D_{2}+\beta_{3} D_{3}+\beta_{4} X_{4}+\beta_{5} X_{5}+u_{i} \\
& Y=\text { variável dependente binária relativa ao aceita ou à recusa das ofertas do jogo } \\
& D_{1}=\text { gênero do proponente (1 para mulher, } 0 \text { para homem) } \\
& D_{2}=\text { gênero do respondente (1 para mulher, } 0 \text { para homem) } \\
& D_{3}=\text { interação entre gêneros ( } 1 \text { se os gêneros foram diferentes e } 0 \text { se eles foram iguais) } \\
& X_{4}=\text { variável escalar para renda do respondente } \\
& X_{5}=\text { variável composta por número de anos (idade) do respondente } \\
& \text { Os resultados dessa regressão são apresentados na Tabela } 10 .
\end{aligned}
$$


Tabela 10

Resultados da Regressão para Aceite ou Recusa das Ofertas por Parte dos Respondentes

\begin{tabular}{lcccc}
\hline Variáveis & Siglas & Odds ratio & $\mathbf{z}$ & P>t \\
\hline gênero do proponent & $\mathrm{D}_{1}$ & .4540 & -1.09 & 0.274 \\
gênero do respondent & $\mathrm{D}_{2}$ & .7036 & -0.49 & 0.623 \\
interação entre gêneros & $\mathrm{D}_{3}$ & .2955 & -1.68 & $\mathbf{0 . 0 9 3}$ \\
Renda & $\mathrm{X}_{1}$ & .4093 & -2.85 & $\mathbf{0 . 0 0 4}$ \\
Idade & $\mathrm{X}_{2}$ & 1.3265 & 2.25 & $\mathbf{0 . 0 2 5}$ \\
Cons & Cons & 1.5834 & 0.17 & 0.867 \\
\hline
\end{tabular}

Nota. R-square $=0,2247 / n=130$. Fonte: Dados da pesquisa.

Os resultados da regressão logística mostraram como significativas as variáveis $\mathrm{D}_{3}, \mathrm{X}_{1}$ e $\mathrm{X}_{2}$. A análise do odds ratio da variável proxy para interação entre os gêneros foi igual a 0.2955 , e, dessa forma, tem-se que, para cada proposta recusada há uma chance de aproximadamente $70 \%$ dessa recusa estar relacionada a uma interação entre gêneros diferentes. Foi observado que o gênero do proponente e do respondente isoladamente não foram apontados como relevantes. Além disso, os resultados mostraram que o aceite das ofertas é mais provável em grupos nos quais a renda do respondente é menor. Quanto ao fator idade, a maior idade do respondente ampliou sua propensão em aceitar a oferta.

Tomando como base os resultados apresentados na Tabela 9, a qual mostrou que o grupo com maior percentual de rejeição foi o $\mathrm{MH}$, e os da Tabela 8, que mostrou que as ofertas médias das mulheres para esse mesmo grupo foram semelhantes às observadas na média geral do Jogo, e somando-se a isso o resultado da regressão logística (Tabela 10) que apontou para o fato de que a interação entre os gêneros estava associada à maior possibilidade de rejeição das ofertas, é possível encontrar subsídios que levam a não aceitação da segunda hipótese desse estudo, que previu uma maior propensão à aceitação de ofertas femininas quando o respondente era homem.

Nesse sentido, observa-se uma propensão à rejeição masculina em relação às decisões do Jogo sabidamente tomadas por mulheres. Tal achado provoca reflexões acerca do papel desempenhado por homens e mulheres no ambiente organizacional, e aponta indícios de que há maior potencial para a geração conflitos entre os gêneros quando as decisões são tomadas por mulheres em razão da maior rejeição masculina, corroborando Gneezy et al. (2003). O resultado da regressão logística confirmou esse achado, uma vez que a variável proxy para a interação entre os gêneros $\left(D_{3}\right)$ mostrou-se estatisticamente significativa para explicar o aceite ou a recusa das ofertas.

Ressalta-se que os jogos experimentais buscam apontar elementos muitas vezes implícitos no processo decisório e não podem ser generalizados para cada indivíduo isoladamente. Camerer (2003) e Damme et al. (2014) lembram que a utilização de Jogos como estratégia metodológica tem como objetivo lançar luz sobre a interação humana em situações de simulação, sendo os jogos entendidos como modelos simplificados da realidade.

Importante ressaltar que a rejeição da segunda hipótese desse estudo não corrobora outros estudos semelhantes conduzidos nos EUA (Eckel \& Grossman, 2001) e no Canadá (Saad \& Gill, 2001), que observaram maior aceite por parte dos homens em relação às ofertas femininas. A cordialidade e os aspectos culturais foram apontados como explicativos para os resultados encontrados nesses países. Resta investigar quais fatores orientaram as decisões observadas para o caso brasileiro, o que exigirá novas pesquisas sobre o tema. 


\section{Discussão dos Resultados}

Kray e Thompson (2005) consideram o JU como um paradoxo de simplicidade e complexidade. De fato, apesar da simplicidade de sua realização, os motivos intrínsecos às respostas dos jogadores permitem muitas inferências e especulações.

Os resultados encontrados no presente estudo suscitam reflexões sobre como as diferenças entre os gêneros podem trazer repercussões para o processo decisório, tendo em vista a perspectiva da economia comportamental. Corroborando os achados de Solnick (2001) e Eckel e Grossman (2001), as mulheres mostraram-se mais generosas em suas ofertas quando comparadas aos homens no JU, o que permitiu aceitar como verdadeira a primeira hipótese proposta para este estudo. Tal achado pode contribuir de diferentes formas para a gestão organizacional, favorecendo a otimização do portfólio das decisões corporativas. Dessa forma, poder-se-ia afirmar que funções permeadas por decisões que exijam maior capacidade de empatia, conciliação ou conservadorismo pareçam mais próximas ao perfil feminino, ao passo que decisões que exijam maior tolerância ao risco e à maximização dos resultados individuais podem ser mais bem trabalhadas por homens. Contudo, é importante lembrar que o gênero por si só não é uma variável definidora, mas apenas uma das que merecem ser consideradas pela gestão organizacional.

A segunda hipótese desse trabalho não foi corroborada, uma vez que foi observado que os homens apresentaram maior propensão à recusa de ofertas femininas em comparação com as ofertas masculinas, ainda que as ofertas femininas tenham sido mais generosas. Tais achados suscitam questões polêmicas relativas à diversidade de gênero, e apontam para a maior possibilidade de que sejam gerados conflitos entre gêneros em grupos de trabalho nos quais os homens desempenhem papel de subordinação em relação às mulheres. Fatores diversos podem ser apontados como explicativos, sejam estes culturais, discriminatórios, relativos aos estereótipos de gênero ou outros; contudo, as limitações deste estudo não permitem apontar quais são eles, o que requer estudos adicionais sobre o tema.

Em síntese, este trabalho oferece pelo menos duas contribuições para a prática da gestão organizacional no Brasil: (a) ao aceitar como verdadeira a primeira hipótese, foi observado que a gestão do processo decisório pode ser beneficiada ao levar em consideração o gênero do tomador de decisão; (b) ao rejeitar a segunda hipótese proposta, observa-se que o gênero do tomador de decisão pode influenciar na forma como a decisão é recebida por aqueles que por ela são afetados, o que aponta para certo potencial de conflito em processos decisórios que envolvam gêneros distintos, notadamente, quando o gênero feminino é o tomador de decisão.

Assim, uma das principais contribuições deste estudo está em observar que tanto o gênero do tomador de decisão (hipótese 1) quanto o daquele que é afetado pela decisão tomada (hipótese 2) mostraram-se influenciadores do processo decisório, sendo esse aspecto merecedor de atenção por parte dos gestores organizacionais.

Análises complementares buscaram evidenciar outras fontes de explicação para os resultados encontrados, tais como a renda e a idade dos jogadores. Foi observado que a renda é fator que influencia tanto o valor a ser ofertado como a decisão entre aceitar ou rejeitar uma proposta. Já a idade somente se mostrou significativa para o aceite das ofertas, mostrando que a maior idade está relacionada à maior disposição em aceitar uma oferta.

Interessante perceber que, mesmo em condição de ofertas consideradas injustas, a recusa não é uma resposta padrão, e que existem indivíduos propensos a sua aceitação. O oposto também foi percebido. Mesmo em circunstâncias de ofertas consideradas generosas, existem pessoas propensas a recusar. Portanto, o que faz as pessoas aceitarem ofertas injustas e recusarem ofertas generosas? De fato, a premissa de maximização da utilidade, da forma como foi prevista pela economia neoclássica, não é capaz de explicar tais eventos em sua totalidade. Outras variáveis devem ser agregadas aos modelos explicativos, e o fator gênero parece ser uma das que merecem consideração. 
As limitações intrínsecas ao experimento econômico como estratégia de pesquisa devem ser ressaltadas. Pesquisas conduzidas em ambiente controlado não podem ser entendidas como protótipos da realidade, uma vez que muitas variáveis existentes e relevantes no mundo real não são reproduzidas em laboratório. $\mathrm{O}$ experimento é, portanto, um modelo de decisão simplificado que busca analisar o comportamento específico dos agentes em condições em que há variáveis manipuladas e a interpretação dos resultados aqui descritos merece cautela, uma vez que também estão sujeitos às especificidades de seu universo amostral.

Por fim, convém lembrar que estudos semelhantes a esse muitas vezes suscitam mais perguntas do que respostas, sendo este também o seu mérito. Este estudo tem a pretensão de motivar novas pesquisas no Brasil, numa abordagem experimental. Tal como afirmaram Güth e Kocher (2014), a compreensão dos fatores que orientam as decisões do JU é algo que tem tomado tempo dos pesquisadores. No Brasil, esse empreendimento está apenas começando.

\section{Referências}

Andreoni, J., \& Vesterlund, L. (2001). Which is the fair sex? Gender differences in altruism. Quarterly Journal of Economic, 116(1), 293-312. http://dx.doi.org/10.1162/003355301556419

Ardila, A., Rosselli, M., \& Inozemtseva, E. M. O. (2011). Gender differences in cognitive development. Developmental Psychology, 47(4), 984-990. http://dx.doi.org/10.1037/a0023819

Ávila, F., \& Bianchi, A. M. (Orgs.). (2015). Guia de economia comportamental e experimental (L. T. Motta \& P. Futagawa, trads., contém partes traduzidas e adaptadas dos guias "The behavioral economics guide 2014" e "The behavioral economics guide 2015"). São Paulo: EconomiaComportamental.org.

Beckmann, D., \& Menkhoff, L. (2008). Will women be women? Analyzing the gender difference among financial experts. International Review of Social Sciences, 61(3), 364-384. http://dx.doi.org/10.1111/j.1467-6435.2008.00406.x

Blau, F. D., \& Kahn, L. M. (2000). Gender differences in pay. Journal of Economic Perspectives, 14(4), 75-99. http://dx.doi.org/10.1257/jep.14.4.75

Camerer, C. (2003). Behavioural game theory: experiments in strategic interaction. Princeton: Princeton University Press.

Camerer, C., \& Thaler R. H. (1995). Anomalies: ultimatums, dictators and manners. Journal of Economic Perspective, 9(2), 209-219. http://dx.doi.org/10.1257/jep.9.2.209

Castillo, M. E., \& Cross, P. J. (2008). Of mice and men: within gender variation in strategic behavior. Games and Economic Behavior, 64(2), 421-432. http://dx.doi.org/10.1016/j.geb.2008.01.009

Charness, G., \& Gneezy, U. (2012). Strong evidence for gender differences for risk taking. Journal of Economic Behavior \& Organization, 83(1), 50-58. http://dx.doi.org/10.1016/j.jebo.2011.06.007

Crawford, V. (2002). Introduction to experimental game theory. Journal of Economic Theory, 104(1), 1-15. http://dx.doi.org/10.1006/jeth.2001.2909

Croson, R., \& Gneezy, U. (2009). Gender differences in preferences. Journal of Economic Literature, 47(2), 448-474. http://dx.doi.org/10.1257/jel.47.2.448

Damme, E. V., Binmore, K. G., Roth, A., Samuelson, E., Winter, E., Bolton, G. E., Dufwewnberg, G. K., Gneezy, M., Kocher, M., Sanfey, A. G., Kliemt, H., Selten, R., Nagel. R., \& Azar, O. (2014). 
How Werner Güth's ultimatum game shaped our understanding of social behavior. Journal of Economic Behavior \& Organization, 108, 292-318. http://dx.doi.org/10.1016/j.jebo.2014.10.014

Dittrich, M. (2015). Gender differences in trust and reciprocity: evidence from a large-scale experiment with heterogeneous subjects. Journal of Applied Economics, 47(36), 3825-3838. http://dx.doi.org/10.1080/00036846.2015.1019036

Eckel, C. C., \& Grossman, P. J. (1998). Are women less selfish than men? Evidence from dictator experiments. The Economic Journal, 108(448), 726-735. http://dx.doi.org/10.1111/14680297.00311

Eckel, C. C., \& Grossman, P. J. (2001). Chivalry and solidarity in ultimatum games. Economic Inquiry, 39(2), 171-188. http://dx.doi.org/10.1111/j.1465-7295.2001.tb00059.x

Eckel, C. C., \& Grossman, P. J. (2008). Men, women and risk aversion: experimental evidence. Handbook of Experimental Economics Results, 1, 1061-1073. http://dx.doi.org/10.1016/S15740722(07)00113-8

Exadaktylos, F., Espín, A. M., \& Brañas-Garza, P. (2013). Experimental subjects are not different. Scientific Reports, 1213(3). http://dx.doi.org/10.1038/srep01213

Fabre, E. F., Causse, M., Pesciarelli, F., \& Cacciari, C. (2015). Sex and the money: how gender stereotypes modulate economic decision-making. An ERP study. Neuropsychologia, 75, 221-232. http://dx.doi.org/10.1016/j.neuropsychologia.2015.06.013

Fabre, E. F., Causse, M., Pesciarelli, F., \& Cacciari, C. (2016). The responder gender stereotypes modulates the strategic decision-making of proposers playing the ultimatum game. Frontiers in Psychology, 7(12), 1-9. http://dx.doi.org/10.3389/fpsyg.2016.00012

García-Gallego, A., Georgantzis, N., \& Jaramillo-Gutiérrez, A. (2012). Gender differences in ultimatum games: despite rather than due to risk attitudes. Journal of Economic Behavior and Organization, 83(1), 42-49. http://dx.doi.org/10.1016/j.jebo.2011.06.012

Gneezy, U., Niederle, M., \& Rustichini, A. (2003). Performance in competitive environments: gender differences. Quarterly Journal of Economics, 118(3), 1049-1074. http://dx.doi.org/10.1162/00335530360698496

Güth, W., \& Kocher, M. G. (2014). More than thirty years of ultimatum bargaining experiments: motives, variations, and a survey of the recent literature. Journal of Economic Behavior and Organization, 108, 396-409. http://dx.doi.org/10.1016/j.jebo.2014.06.006

Güth, W., Schmittberger, R., \& Schwarze, B. (1982). An experimental analysis of ultimatum bargaining. Journal of Economic Behavior and Organization, 3(4), 367-388. http://dx.doi.org/10.1016/01672681(82)90011-7

Henrich, J., Boyd, R., Bowles, S., Camerer, E. F., Gintis, H., \& McElreath, R. (2001). In search of homo economicus: behavioral experiments in 15 small-scale societies. The American Economic Review, 91(2), 73-78. http://dx.doi.org/10.1257/aer.91.2.73

Kahneman, D., Knetsch, J. L., \& Thaler, R. H. (1986b). Fairness and the assumptions of economics. Journal of Business, 59(4), S285-S300. http://dx.doi.org/10.1086/296367

Kahneman, D., Knetsch, J. L., \& Thaler, R. (1986a). Fairness as a constraint on profit seeking: entitlements in the market. The American Economic Review, 76(4), 728-741.

Kahneman, D., \& Tversky, A. (1979). Prospect theory: an analysis of decision under risk. Econometrica, 47(2), 263-292. http://dx.doi.org/10.2307/1914185 
Kray, L. J., \& Thompson, L. (2005). Gender stereotypes and negotiation performance: an examination of theory and research. Research in Organizational Behavior, 26, 103-182. http://dx.doi.org/10.1016/S0191-3085(04)26004-X

Maltz, D. N., \& Borker, R. (1982). A cultural approach to male-female miscommunication. In J. J. Gumpertz (Ed.), Language and social identity (pp. 168-185). Malden, MA: Cambridge University Press.

McGee, P., \& Constantinides, S. (2013). Repeated play and gender in the ultimatum game. Journal of Socio-Economics, 42, 121-126. http://dx.doi.org/10.1016/j.socec.2012.11.012

Meier-Pesti, K., \& Penz, E. (2008). Sex or gender? Expanding the sex-based view by introducing masculinity and femininity as predictors of financial risk taking. Journal of Economic Psychology, 29(2), 180-196. http://dx.doi.org/10.1016/j.joep.2007.05.002

Mulac, A., Bradac, J., \& Gibbons, P. (2001). Empirical support for the gender as culture hypothesis. Human Communication Research, 27(1) 121-152. http://dx.doi.org/10.1111/j.14682958.2001.tb00778.x

Ortmann, A., \& Tichy, L. (1999). Gender effects in the laboratory: evidence from prisoners' dilemma games. Journal of Economic Behavior and Organization, 39(3), 327-339. http://dx.doi.org/10.1016/S0167-2681(99)00038-4

Prime, J. L., Carter, N. M., \& Welbourne, T. M. (2009). Women "take care," men "take charge": managers' stereotypic perceptions of women and men leaders. The Psychologist-Manager Journal, 12(1), 25-49. http://dx.doi.org/10.1080/10887150802371799

Rapoport, A., \& Chammah, A. M. (1965). Sex differences in factors contributing to the level of cooperation in the prisoner's dilemma game. Journal of Personality and Social Psychology, 2(6), 831-838. http://dx.doi.org/10.1037/h0022678

Roth, A. E., Prasnikar, V., Fujiwara, M., \& Zamir, S. (1991). Bargaining and market behavior in Jerusalem, Ljubljana, Pittsburgh, and Tokyo: an experimental study. The American Economic Review, 81(5), 1068-1095.

Saad, G., \& Gill, T. (2001). Sex differences in the ultimatum game. Journal of Bioeconomics, 3(2), 171193. http://dx.doi.org/10.1023/A:1020583425623

Solnick, S. J. (2001). Gender differences in the ultimatum game. Economic Inquiry, 39(2), 189-200. http://dx.doi.org/10.1111/j.1465-7295.2001.tb00060.x

Straits, B. C., \& Singleton, R. (2010). Approaches to social research (5th ed.). New York: Oxford University Press.

Sutter, M., Bosman, R., Kocher, M., \& Winden, F. van (2009). Gender pairing and bargaining: beware the same sex. Experimental Economics, 12(3), 318-331. http://dx.doi.org/10.1007/s10683-0099217-9

Svorenčík, A., \& Maas, H. (Eds.). (2015). The making of experimental economics: witness seminar on the emergence of a field. Switzerland: Springer International Publishing.

Weiss, E. M., Kemmlera, G., Deisenhammerb, E. A., Fleischhackera, W. W., \& Delazer, M. (2003). Sex differences in cognitive functions. Personality and Individual Differences, 35(4), 863-875. http://dx.doi.org/10.1016/S0191-8869(02)00288-X

Zak, P. J., Kurzban, R., Ahmadi, S., Swerdloff, R. S., Park, J., Efremidze, L., Redwine, K., Morgan, K., \& Matzner, W. (2009). Testosterone administration decreases generosity in the ultimatum game. Plos One, 4(12), 1-8. http://dx.doi.org/10.1371/journal.pone.0008330 


\section{Dados dos Autores}

\section{Angela Cristiane Santos Póvoa}

Rua Imaculada Conceição, 1155, Prado Velho, 80215-901, Curitiba, PR, Brasil. E-mail: angelapovoa@ gmail.com

Maickel Robert Maffezzolli

Rua Imaculada Conceição, 1155, Prado Velho, 80215-901, Curitiba, PR, Brasil. E-mail: maickel.maffezzolli@gmail.com

Wesley Pech

429, North Church Spartanburg, 29303, Spartanburg, SC, EUA. E-mail: pechwj@wofford.edu

Wesley Vieira da Silva

Rua Imaculada Conceição, 1155, Prado Velho, 80215-901, Curitiba, PR, Brasil. E-mail: wesley.vieira@pucpr.br 